\title{
The impact of serum uric acid on the natural history of glomerular filtration rate: a retrospective study in general population
}

Ying $\mathrm{Xu}$, Xiang Liu, Xiaohe Sun, Yibing Wang

Serum uric acid (SUA) level has been proposed to have important connections with chronic kidney disease (CKD), while the impact of SUA level on the natural history of glomerular filtration rate (GFR) decline remains unknown. The present study aims to study the association of the SUA level with the GFR decline in a general population. 2789 subjects who visited the Health Checkup Clinic both at 2008 and 2013 were identified. A significant inverse correlation was observed between change in SUA from 2008 to 2013 (DSUA) and change in eGFR ( $\triangle \mathrm{eGFR}$ ) during the same period. Multivariate regression analysis of $\triangle \mathrm{eGFR}$ indicated that the increase in SUA over time were negative predictor of the change in eGFR. Our result indicates that the decline of eGFR over years is larger in subjects with an increased SUA level, which helps to underline the importance of SUA level management in the context of kidney function preservation. 
1 The impact of serum uric acid on the natural history of glomerular filtration rate: a

2 retrospective study in general population

3

4 Ying $\mathrm{Xu}^{1}$, Xiang Liu ${ }^{1}$, Xiaohe Sun ${ }^{2}$, Yibing Wang ${ }^{3 \#}$

5

6 From ${ }^{1}$ Department of Nephrology, Shandong Provincial Hospital Affiliated to

7 Shandong University, Jinan, China;

8 2School of Medicine, Shandong University, Jinan, China;

9 3Department of Aesthetic Plastic and Burn Surgery, Shandong Provincial Hospital

10 Affiliated to Shandong University, Jinan China; Health Checkup Clinic, Shandong

11 Provincial Hospital Affiliated to Shandong University, Jinan China;

12

13 \#To whom correspondence should be addressed. Yibing Wang, Department of

14 Aesthetic Plastic and Burn Surgery, Shandong Provincial Hospital Affiliated to

15 Shandong University Jinan, China, Tel.: +86 531 68776557; Fax: +86 531

16 7909629. E-mail address: wyb0616@163.com 


\section{Abstract}

19 Serum uric acid (SUA) level has been proposed to have important connections

20 with chronic kidney disease (CKD), while the impact of SUA level on the natural

21 history of glomerular filtration rate (GFR) decline remains unknown. The present

22 study aims to study the association of the SUA level with the GFR decline in a

23 general population. 2789 subjects who visited the Health Checkup Clinic both at

242008 and 2013 were identified. A significant inverse correlation was observed

25 between change in SUA from 2008 to 2013 ( $\triangle$ SUA) and change in eGFR

26 ( $\triangle$ eGFR ) during the same period. Multivariate regression analysis of $\Delta$ eGFR

27 indicated that the increase in SUA over time were negative predictor of the

28 change in eGFR. Our result indicates that the decline of eGFR over years is larger

29 in subjects with an increased SUA level, which helps to underline the importance

30 of SUA level management in the context of kidney function preservation. 


\section{1.Introduction}

33 Uric acid (UA) is a poorly soluble end product of purine nucleotides degradation,

34 and hyperuricemia is defined as serum uric acid (SUA) level $>420 \mathrm{umol} / \mathrm{L}(7 \mathrm{mg} / \mathrm{dl}$ )

35 in males and $>360 \mathrm{umol} / \mathrm{L}$ ( $>6 \mathrm{mg} / \mathrm{dl}$ ) in females (Chuang et al., 2012). Recent

36 researches have suggested SUA to be an independent risk factor for

37 cardiovascular disease (Chuang et al., 2012) and metabolic syndrome (MetS) (Liu

38 et al., 2014; Nejatinamini et al., 2015). Hyperuricemia was also suggested to be

39 associated with the onset of type 2 diabetes mellitus (DM) (Miyake et al., 2014)

40 and stroke (Qin et al., 2014).

41 As long as the kidney is concerned, the pathophysiological role of SUA in the

42 chronic kidney disease (CKD) has attracted a lot of nephrologists and there are

43 many studies published. It has been established that in patients with gout and

44 acute UA nephropathy, the treatment of hyperuricemia is of clinical benefits. For

45 patients without gout and acute nephropathy, the first aspect is the relationship

46 between hyperuricemia and CKD progression. Whether SUA is an independent

47 risk factor or only a marker of the progression of CKD is still a matter of debate. In

48 the past, hyperuricemia was thought to be simply a reflection of kidney damage

49 because it was assumed that a decrease in kidney blood flow causes the

50 decrease in UA clearance. However, high uric acid levelswas reported to be

51 associated with increased rates of glomerular filtration rate (GFR) decline in

52 multiple cross sectional studies in CKD patients (Ben-Dov and Kark, 2011;

53 Satirapoj et al., 2010). On the other hand, an association of SUA with CKD was

54 not demonstrated in other studies. The elevation of SUA did not contribute to the

55 progression of CKD in the Mild to Moderate Kidney Disease (MMKD) study, which

56 only recruited non-diabetic patients (Sturm et al., 2008). The second aspect is

57 whether hyperuricemia is a predictor of incidence of CKD. There was no

58 significant association between SUA and the incidence of CKD during the 5-year

59 follow-up in the Cardiovascular Health Study, which recruited 5808 subjects

60 (Chonchol et al., 2007). However, in type $2 \mathrm{DM}$, hyperuricemia has been reported

61 to be correlated with the onset of proteinuria (Tseng, 2005) and renal dysfunction

62 (Bo et al., 2001; De Cosmo et al., 2015).. A recent meta-analysis that included 13 
63 studies also reported that a relationship between increasing SUA level and new

64 onset of CKD (defined by eGFR $<60 \mathrm{~mL} / \mathrm{min} / 1.73 \mathrm{~m}^{2}$ ) (Li et al., 2014).

65 While the conclusion is still uncertain, most studies were done study the 66 relationship of hyperuricemia and CKD incidence/progression, it also remains

67 unknown whether the SUA level is associated with the natural decline of GFR with

68 aging. It is of importance to study the strategies that could prevent the incidence

69 of CKD and preserve the GFR in general population. The present study aims to

70 study the association of the SUA level with the GFR decline in a general

71 population who visited the Health Checkup Clinic regularly, and our results

72 suggested a role of SUA level in the GFR decline.

73 


\section{$74 \quad$ 2.Methods}

75 The protocol of this study was approved by the Clinical Investigation Ethics

76 Committee of Shandong Provincial Hospital Affiliated Shandong Provincial

77 Hospital (Reference No.2015-040) and was conducted in strict adherence with

78 the principles of the Declaration of Helsinki.

2.1. Study population:

81 This is a retrospective study. Computer-based data of Health Checkup Clinic,

82 Shandong Provincial Hospital Affiliated to Shandong University (Jinan, China)

83 were available from Jan 1st, 2008 through Dec 31st, 2013. Subjects who visited

84 the Heath Checkup at 2008 and 2013 were identified using ID numbers,

85 birthdates and other identifiers. All subjects are $>18$ years old. This study

86 excludes outpatient or clinical patients. Patients with acute kidney injury,

87 amputation, heart failure, severe liver disease, infection disease, malignant

88 disease and pregnancy were excluded. To confirm the accuracy, all the matches

89 were visually inspected. Thus, we identified 2789 adults, of which 205 subjects

90 were excluded for incomplete records. This left 2584 subjects (1791 males and

91793 females) for analysis. All subjects gave the oral consent that the Health

92 Checkup Clinic of Shandong Provincial Hospital could use the anonymous data

93 for research purpose only when they visited at the first time.

94 In 2008 and 2013, all subjects completed a detailed questionnaire

95 administered by trained interviewers on life style factors such as smoking, alcohol

96 consuming, and physical activity. Weight and height were measured with light

97 indoor clothes and without shoes. An automated sphygmomanometer was used

98 to measure systolic and diastolic blood pressures (SBP/DBP) with subjects in the

99 seated position in triplicate. A blood sample was taken after an overnight fast.

100 Biomedical parameters including serum uric acid, serum creatinine (Scr), fasting

101 plasma glucose (FPG) and lipids were measured using a biochemical auto-

102 analyzer in the Clinical Laboratory of the Shandong Provincial Hospital affiliated to

103 Shandong University using standardized procedures. 
104 2.2. Definition:

105 Body mass index was calculated as weight $(\mathrm{kg})$ divided by the square of the

106 height $(m)$. Estimated glomerular filtration rate (eGFR) was calculated using

107 Modification of Diet in Renal Disease (MDRD) formula for Chinese (Kong et al.,

108 2013; Ma et al., 2006). According to a research published in 2013 that recruited

109682 patients and 295 healthy adults,, MDRD formula for Chinese is comparable to

110 the CKD-EPI two-level race equation. They both performed better than the MDRD

111 Study equation and the CKD-EPI four-level equation (Kong et al., 2013). The

112 longitudinal eGFR changes ( $\triangle$ eGFR) were calculated as the eGFR at 2013

113 minus the eGFR at 2008 (eGFR13 - eGFR08). Hyperuricemia is defined as

114 serum uric acid level $>420 \mathrm{umol} / \mathrm{L}(7 \mathrm{mg} / \mathrm{dl})$ in males and $>360 \mathrm{umol} / \mathrm{L}(>6 \mathrm{mg} / \mathrm{dl})$

115 females (Chuang et al., 2012).

116 2.3. Data analysis:

117 Statistical analysis was conducted by using SAS, version 9.4) (SAS Institute Inc).

118 The graph was drafted using SPSS, version 22. Values of $P<0.05$ were

119 considered to be statistically significant. Kolmogorov-Smirnov test was used for

120 normality distribution.Descriptive statistics are presented as means with standard

121 deviations for normally distributed data, median with interquartile range (Q75,

122 Q25) for non-normally distributed data, and frequencies with percentage for

123 categorical data. Paired t-test were used for normally distributed continuous

124 variables, while Wilcoxon Signed-Rank test were used for non-normally

125 distributed data in comparison between data in year of 2008 and that in 2013.

126 Univariate and multivariatelinear regression analysis was employed to estimate

127 the relationship between SUA level and eGFR change over 5 years. 


\section{3.Results}

130 3.1. Demographic characteristics.

131 Table 1 shows the demographic characteristics in the cohort. The SUA level was

132 significantly increased from year 2008 to 2013 (322.00(382.00, 267.00) versus

$133352.00(410.00,295.00)$ umol/L, p<0.01). While the decline of eGFR across the 5-

134 year period was statistically significant $(103.99(113.31,95.11)$ to $101.19(113.24$,

$135101.19) \mathrm{mL} / \mathrm{min} / 1.73 \mathrm{~m}^{2}, \mathrm{p}=0.08$ ) but in a very small mannor There were no

136 differences in the BMI, systolic blood pressure (BP). Fasting plasma glucose

137 (FPG) , serum total cholesterol (TC) and serum triglycerides (TG) were slightly

138 increased in 2013 compared to 2008, and the diastolic BP was marginally

139 decreased. The ratio of subjects with dipstick-positive proteinuria at 2008 and

1402013 was $3.4 \%$ and $2.8 \%$ respectively

142 3.2. Relationship between longitudinal changes in SUV and eGFR.

143 Univariate linear regression analysis did not indicate a significant correlation

144 between baseline SUA at 2008 and the change in eGFR from 2008 to 2013

$145(\Delta$ eGFR $)(r=0.03, p=0.09)$. In contrast, a significant inverse correlation was

146 observed between change in SUA from 2008 to 2013 ( $\Delta$ SUV) and $\Delta$ eGFR during

147 the same period as shown in Figure $1(r=-0.37, p<0.01)$.

148 As listed in Table 2, gender, fasting plasma glucose (FPG), serum total

149 cholesterol (TC), serum triglycerides (TG) and baseline eGFR were significant

150 predictors of the $\triangle$ eGFR $\left(t^{\prime}=8.30, r=0.15, r=0.10, r=0.09, r=-0.30\right.$, respectively; $p<$

151 0.01). Drink $(p=0.95)$ or smoke habit $(p=0.98)$, BMI $(p=0.42)$, systolic blood

152 pressure (SBP) $(p=0.87)$, and diastolic blood pressure (DBP) $(p=0.52)$ were not

153 associated with $\triangle$ eGFR.

155 3.3. Determinants of longitudinal changes in eGFR ( $\triangle$ eGFR) in people with

156 increased SUA level and decreased SUA level over 5 years

157 . Secondary analyses included a trichotomized variable defined by subject's SUA

158 increase versus decline from year 2008 to 2013 . For a changed SUA level $<10$ 
159 umol/L, either increased or decreased, it is not clinically meaningful since it could

160 be the experiment error. So we divided the subjects into three groups: subjects

161 with increased $\operatorname{SUA}(\triangle \mathrm{SUA}>10 \mathrm{umol} / \mathrm{L})$, those with unchanged SUA (-10

$162 \mathrm{umol} / \mathrm{L}<\triangle \mathrm{SUA}<10 \mathrm{umol} / \mathrm{L}$ ), and those with decreased SUA ( $\triangle \mathrm{SUA}<-10 \mathrm{umol} / \mathrm{L}$ ).

163 The demographic characteristics of thress groups were shown in Table 3. There

164 were no differences in the distribution of gender, age, drink or smoke habit

165 between threegroups (all p-value $>0.05$ ). The baseline SUA is lower in the

166 Increased SUA group. Together with SUA level, BMI, DBP, FPG and TG, , were

167 ower in the Increased SUA group compared with the other two groups. eGFR of

168 the Increased SUA group was highest. This indicates that the Increased SUA was

169 at a "healthier" state at 2008. However, after 5 years, the eGFR of the Increased

170 SUA group declined by $5.26(3.71,-12.44) \mathrm{mL} / \mathrm{min} / 1.73 \mathrm{~m}^{2}$, and the eGFR of the

171 Decreased SUA group incremented by3.23(15.25, -4.63$) \mathrm{mL} / \mathrm{min} / 1.73 \mathrm{~m}^{2}$ with a $\mathrm{p}$ -

172 value $<0.01$

173

174 Based on the result of univariate linear regression analysis and the

175 comparison between the three groups, we identified the independent variables

176 that were put into multivariate linear regression model. They meet both criteria,

177 first, $p$ of urivariate linear regression analysis $<0.1$, and second, there were

178 differences among three groups. Together with the trichotomized variable defined

179 by subject's SUA change, baseline SUA, FPG, TC, TG, and baseline eGFR were

180 fitted in the multiple linear regression model. The colinearity diagnostics show that

181 vif was close to 0 , and condition index was $>10$, indicating that multicollinearity

182 exists. However, there were no closely related variables were detected. Using

183 forward stepwise selection with inclusion criteria of $p<0.05$ and exclusion criteria

184 of $p>0.10$, we get the multivariate regression analysis results as shown in Table 4.

185 Multivariate regression analysis indicated that increased SUA together with

186 baseline SUA level, baseline FPG, baseline TC, baseline TG, and baseline eGFR

187 were negative predictor of $\triangle$ eGFR ( $\triangle$ eGFR = eGFR at 2013 - eGFR at 2008)

188 with $\mathrm{R}^{2}$ of 0.17 .

189 
190 Discussion

191 The present study demonstrates that the change in SUA during a 5-year period is

192 inversely associated with the decline of eGFR during the same period.

193 Chronic kidney disease (CKD) has become a global public health problem

194 because of the high prevalence and the accompanying increase in the risk of end

195 stage renal disease (ESRD), cardiovascular disease and premature death (Tonelli

196 et al., 2006). There are many cohorts that show the development and progression

197 of CKD are linked with uric acid level. A Korean study showed that asymptomatic

198 hyperuricimia men had a greater odd (odds ratio, 1.96) of developing CKD than

199 normouricemic men (Ryoo et al., 2013). Epidemiologic data from a Japanese

200 cohort showed that hyperuricemia was an independent risk factor for decrease in

201 eGFR (Iseki et al., 2013). New-onset of microalbuminuria was shown to be

202 associated with hyperuricemia in a middle-age and elderly Taiwanese population

203 (Chang et al., 2013). However, the highest uric acid level did not lead to an

204 increased incidence of CKD according to an analysis of the MDRD Study

205 population (Madero et al., 2009). In contrast, effects of hyperuricemia on a natural

206 history of GFR have been less examined. In the present study, we did find a

207 difference in the decline of eGFR over 5 years between asymptomatic

208 hyperuricemia subjects and normal SUA level subjects (data not shown). While a

209 change in SUA is associated with the $\triangle$ eGFR, though an association could not

210 indicate cause-effect relationship. Our results are in consistent with a recent study,

211 which included two cohorts, urban residents and rural town residents respectively.

212 Their results also indicate that the elevation of SUA accelerates the yearly decline

213 in eGFR (Akasaka et al., 2014).

214 Interventions targeting at hyperuricemia may decrease disease progression in

215 patients with CKD. In a trial of 96 patients with CKD stages 3 to 4 with mean

216 eGFR of $44.62 \pm 14.38 \mathrm{~mL} / \mathrm{min} / 1.73 \mathrm{~m}^{2}$, allopurinol or control was randomly

217 assigned. Those patients with lower serum uric acid levels by the use of

218 allopurinol had an increase in eGFR of $3.3 \pm 1.2 \mathrm{~mL} / \mathrm{min} / 1.73 \mathrm{~m}^{2}$ per year,

219 whereas eGFR decreased $1.3 \pm 0.6 \mathrm{~mL} / \mathrm{min} / 1.73 \mathrm{~m}^{2}$ in the control group during a 
220 12-month follow-up ( $\mathrm{P}<0.04)$ (Sezer et al., 2014). In another double-blind,

221 randomized, placebo-controlled single center trial with CKD stage 3-4 patients, 93

222 patients with hyperruicemia were randomized to febuxostat or placebo group. The

223 eGFR of febuxostat group increased nonsignificantly from $31.5 \pm 13.6$ (SD) to

$22433.7 \pm 16.6 \mathrm{~mL} / \mathrm{min} / 1.73 \mathrm{~m}^{2}$ at 6 months. Placebo group showed a significant

225 decrease of eGFR from $32.6 \pm 11.6$ to $28.2 \pm 11.5 \mathrm{~mL} / \mathrm{min} / 1.73 \mathrm{~m}^{2}(P<0.003)$

226 (Sircar et al., 2015). Thus, lowering serum uric acid level could slow the decline in 227 eGFR in CKD stages and 4 patients.

228 In contrast with studies that were done in patients who already have chronic 229 kidney disease, our results show that in health check-up individuals, the increase

230 in SUA level is associated with a greater decline in GFR in a 5-year period. This is 231 in consistent with previous studies that show lowering uric acid level also could 232 preserve GFR in individuals with normal kidney function. Serum uric acid level $233>480 \mathrm{umol} / \mathrm{L}(8 \mathrm{mg} / \mathrm{dL})$ or $<300 \mathrm{umol} / \mathrm{L}(5 \mathrm{mg} / \mathrm{dL})$ was found to be linked with a 234 subsequent increased risk for kidney failure within 2 years (2.9-fold in men and 23510.0 fold in women) in a study of 6,400 individuals with normal kidney function 236 (Iseki et al., 2001). Another 3-months of prospective study showed that lowering 237 serum uric acid level in patients with normal kidney function could preserve eGFR 238 (Kanbay et al., 2007). Taken together, a beneficial effect of controlling SUA level 239 in general asymptomatic population is worthy investigated further.

240 In our study, we focused on the relationship between SUA level changes and 241 eGFR changes, while the predictive value of hyperuricemia on the incidence of 242 CKD or cardiovascular disease (CVD) was not explored. SUA as a risk factor for 243 the incidence of CVD was clearly shown by two recent meta analysis (Braga et al., 244 2016; Li et al., 2016). In one residential cohort, SUA and eGFR was separated 245 assessed as risk factors for CVD. SUA level was shown to be a predictor of 246 incidence of CVD while eGFR was not a predictor (Puddu et al., 2014). Since the 247 incidence of CVD is higher in CKD especially ESRD patients, the impact of SUA 248 change and eGFR change on the incidence of CVD need further studied.

249 The mechanisms that uric acid leads to CKD progression are not fully 250 understood. Uric acid crystals are able to adhere to the renal epithelial cells, 
251 which could induce an acute inflammatory response (Umekawa et al., 2003).

252 Besides the increased risk of kidney stone formation, uric acid adherence has

253 been shown to reduce the GFR decades ago (Spencer et al., 1976). Previous

254 studies demonstrated the mechanisms by which that uric acid damages renal

255 function include activation of cytoplasmic phospholipase A2, inflammatory

256 transcription factor nuclear factor-kb (NF-kB) (Han et al., 2007)as well as

257 upregulation of the systemic cytokine production, such as tumor necrosis factor $\alpha$

258 (Netea et al., 1997), and the local expression of chemokines, such as monocyte

259 chemotactic protein 1 (Kang et al., 2002). Increasing uric acid levels also could

260 induce oxidative stress and endothelial dysfunction.

261 There are limitations in our study. The major limitation of the present study is 262 that our study subjects were not randomly sampled. Thus, some selection bias

263 cannot be excluded in the cohort. This might explain that some continuous

264 variables in our study are not normally distributed. Second, there are no

265 information on medications used, such as uricosuric drugs, xanthine oxidase, and

266 anti-hypertension drugs. So there are maybe some other confounding factors that

267 could not detected by our analysis. Third, some subjects showed increased or

268 decreased eGFR that is greater than $50 \mathrm{~mL} / \mathrm{min} / 1.73 \mathrm{~m}^{2}$ from 2008 t0 2013

269 (Figure 1), which might reflect undetected renal disease. Finally, this study didn't

270 account for all the factors that might have impact on $\triangle$ eGFR such as treatment.

271 In conclusion, in this retrospective study, we observed that larger decline of

272 eGFR over years is associated with the increased SUA level in general

273 population. Together with more causal mechanisms to be discovered, our findings

274 help to underline the importance of SUA level management in the context of

275 kidney function preservation.

276

277 
278 Acknowledgments

279 We thank Shumei Wang and Mingyu Luo for the assistance in statistical analysis.

280 


\section{References}

282 Akasaka, H., Yoshida, H., Takizawa, H., Hanawa, N., Tobisawa, T., Tanaka, M., Moniwa, 283 N., Togashi, N., Yamashita, T., Kuroda, S. Ura, N., Miura, T., Boreas-Ckd Investigators. 284 (2014). The impact of elevation of serum uric acid level on the natural history of 285 glomerular filtration rate (GFR) and its sex difference. Nephrol Dial Transplant 29, 286 1932-1939.

287 Ben-Dov, I.Z., and Kark, J.D. (2011). Serum uric acid is a GFR-independent long-term 288 predictor of acute and chronic renal insufficiency: the Jerusalem Lipid Research Clinic cohort study. Nephrol Dial Transplant 26, 2558-2566.

Bo, S., Cavallo-Perin, P., Gentile, L., Repetti, E., and Pagano, G. (2001). Hypouricemia and hyperuricemia in type 2 diabetes: two different phenotypes. Eur J Clin Invest 31, 318-321.

Braga, F., Pasqualetti, S., Ferraro, S., and Panteghini, M. (2016). Hyperuricemia as risk factor for coronary heart disease incidence and mortality in the general population: a systematic review and meta-analysis. Clin Chem Lab Med 54, 7-15.

Chang, H.Y., Lee, P.H., Lei, C.C., Tung, C.W., Hsu, Y.C., Huang, T.J., Lu, L.C., and Lin, C.L. (2013). Hyperuricemia is an independent risk factor for new onset microalbuminuria in a middle-aged and elderly population: a prospective cohort study in taiwan. PLoS One 8, e61450.

Chonchol, M., Shlipak, M.G., Katz, R., Sarnak, M.J., Newman, A.B., Siscovick, D.S., Kestenbaum, B., Carney, J.K., and Fried, L.F. (2007). Relationship of uric acid with progression of kidney disease. Am J Kidney Dis 50, 239-247.

Chuang, S.Y., Chen, J.H., Yeh, W.T., Wu, C.C., and Pan, W.H. (2012). Hyperuricemia and increased risk of ischemic heart disease in a large Chinese cohort. Int J Cardiol 154, 316-321.

De Cosmo, S., Viazzi, F., Pacilli, A., Giorda, C., Ceriello, A., Gentile, S., Russo, G., Rossi, M.C., Nicolucci, A., Guida, P., Feig, D., Johnson, R. J., Pontremoli, R., A. MD-Annals Study Group. , (2015). Serum Uric Acid and Risk of CKD in Type 2 Diabetes. Clin J Am Soc Nephrol 10, 1921-1929.

310

311

312

313

314

315

316

317

318

319

320

321

322

323

324

325 Han, H.J., Lim, M.J., Lee, Y.J., Lee, J.H., Yang, I.S., and Taub, M. (2007). Uric acid inhibits renal proximal tubule cell proliferation via at least two signaling pathways involving PKC, MAPK, cPLA2, and NF-kappaB. Am J Physiol Renal Physiol 292, F373-381.

Iseki, K., Iseki, C., and Kinjo, K. (2013). Changes in serum uric acid have a reciprocal effect on eGFR change: a 10-year follow-up study of community-based screening in Okinawa, Japan. Hypertens Res 36, 650-654.

Iseki, K., Oshiro, S., Tozawa, M., Iseki, C., Ikemiya, Y., and Takishita, S. (2001). Significance of hyperuricemia on the early detection of renal failure in a cohort of screened subjects. Hypertens Res 24, 691-697.

Kanbay, M., Ozkara, A., Selcoki, Y., Isik, B., Turgut, F., Bavbek, N., Uz, E., Akcay, A., Yigitoglu, R., and Covic, A. (2007). Effect of treatment of hyperuricemia with allopurinol on blood pressure, creatinine clearence, and proteinuria in patients with normal renal functions. Int Urol Nephrol 39, 1227-1233.

Kang, D.H., Nakagawa, T., Feng, L., Watanabe, S., Han, L., Mazzali, M., Truong, L., Harris, R., and Johnson, R.J. (2002). A role for uric acid in the progression of renal disease. J Am Soc Nephrol 13, 2888-2897. 
326 Kong, X., Ma, Y., Chen, J., Luo, Q., Yu, X., Li, Y., Xu, J., Huang, S., Wang, L., Huang, W. 327 Wang, M., Xu, G., Zhang, L., Zuo, L., Wang, H., Chinese e, G.F.R. Investigation 328 Collaboration.(2013). Evaluation of the Chronic Kidney Disease Epidemiology 329 Collaboration equation for estimating glomerular filtration rate in the Chinese population. Nephrol Dial Transplant 28, 641-651. Li, L., Yang, C., Zhao, Y., Zeng, X., Liu, F., and Fu, P. (2014). Is hyperuricemia an independent risk factor for new-onset chronic kidney disease?: A systematic review and meta-analysis based on observational cohort studies. BMC Nephrol 15, 122.

Li, M., Hu, X., Fan, Y., Li, K., Zhang, X., Hou, W., and Tang, Z. (2016). Hyperuricemia and the risk for coronary heart disease morbidity and mortality a systematic review and dose-response meta-analysis. Sci Rep 6, 19520.

Liu, M., He, Y., Jiang, B., Wu, L., Yang, S., Wang, Y., and Li, X. (2014). Association between Serum Uric Acid Level and Metabolic Syndrome and Its Sex Difference in a Chinese Community Elderly Population. Int J Endocrinol 2014, 754678.

Ma, Y.C., Zuo, L., Chen, J.H., Luo, Q., Yu, X.Q., Li, Y., Xu, J.S., Huang, S.M., Wang, L.N., Huang, W., Wang, M., Xu, G.B., Wang, H. Y.(2006). Modified glomerular filtration rate estimating equation for Chinese patients with chronic kidney disease. J Am Soc Nephrol 17, 2937-2944.

Madero, M., Sarnak, M.J., Wang, X., Greene, T., Beck, G.J., Kusek, J.W., Collins, A.J., Levey, A.S., and Menon, V. (2009). Uric acid and long-term outcomes in CKD. Am J Kidney Dis 53, 796-803.

Miyake, T., Kumagi, T., Furukawa, S., Hirooka, M., Kawasaki, K., Koizumi, M., Todo, Y., Yamamoto, S., Abe, M., Kitai, K. Matsuura, B., Hiasa, Y. (2014). Hyperuricemia is a risk factor for the onset of impaired fasting glucose in men with a high plasma glucose level: a community-based study. PLoS One 9, e107882.

351 Nejatinamini, S., Ataie-Jafari, A., Qorbani, M., Nikoohemat, S., Kelishadi, R., Asayesh, 352 H., and Hosseini, S. (2015). Association between serum uric acid level and metabolic syndrome components. J Diabetes Metab Disord 14, 70.

Netea, M.G., Kullberg, B.J., Blok, W.L., Netea, R.T., and van der Meer, J.W. (1997). The role of hyperuricemia in the increased cytokine production after lipopolysaccharide challenge in neutropenic mice. Blood 89, 577-582.

358

359

360

361

362

363

364

365

366 Puddu, P.E., Bilancio, G., Terradura Vagnarelli, O., Lombardi, C., Mancini, M., Zanchetti, A., and Menotti, A. (2014). Serum uric acid and eGFR_CKDEPI differently predict long-term cardiovascular events and all causes of deaths in a residential cohort. Int J Cardiol 171, 361-367.

Qin, L., Yang, Z., Gu, H., Lu, S., Shi, Q., Xing, Y., Li, X., Li, R., Ning, G., and Su, Q. (2014). Association between serum uric acid levels and cardiovascular disease in middleaged and elderly Chinese individuals. BMC Cardiovasc Disord 14, 26.

Ryoo, J.H., Choi, J.M., Oh, C.M., and Kim, M.G. (2013). The association between uric acid and chronic kidney disease in Korean men: a 4-year follow-up study. J Korean Med Sci 28, 855-860.

367

368

369

370 Satirapoj, B., Supasyndh, O., Nata, N., Phulsuksombuti, D., Utennam, D., Kanjanakul, I., Choovichian, P., and Duangurai, K. (2010). High levels of uric acid correlate with decline of glomerular filtration rate in chronic kidney disease. J Med Assoc Thai 93 Suppl 6, S65-70.

371 Sezer, S., Karakan, S., Atesagaoglu, B., and Acar, F.N. (2014). Allopurinol reduces 
372 cardiovascular risks and improves renal function in pre-dialysis chronic kidney 373 disease patients with hyperuricemia. Saudi J Kidney Dis Transpl 25, 316-320.

374 Sircar, D., Chatterjee, S., Waikhom, R., Golay, V., Raychaudhury, A., Chatterjee, S., and 375 Pandey, R. (2015). Efficacy of Febuxostat for Slowing the GFR Decline in Patients 376 With CKD and Asymptomatic Hyperuricemia: A 6-Month, Double-Blind, Randomized, 377 Placebo-Controlled Trial. Am J Kidney Dis 66, 945-950.

378 Spencer, H.W., Yarger, W.E., and Robinson, R.R. (1976). Alterations of renal function 379 during dietary-induced hyperuricemia in the rat. Kidney Int 9, 489-500.

380 Sturm, G., Kollerits, B., Neyer, U., Ritz, E., Kronenberg, F., and Group, M.S. (2008). Uric 381 acid as a risk factor for progression of non-diabetic chronic kidney disease? The 382 Mild to Moderate Kidney Disease (MMKD) Study. Exp Gerontol 43, 347-352.

383 Tonelli, M., Wiebe, N., Culleton, B., House, A., Rabbat, C., Fok, M., McAlister, F., and 384 Garg, A.X. (2006). Chronic kidney disease and mortality risk: a systematic review. J 385 Am Soc Nephrol 17, 2034-2047.

386 Tseng, C.H. (2005). Correlation of uric acid and urinary albumin excretion rate in 387 patients with type 2 diabetes mellitus in Taiwan. Kidney Int 68, 796-801.

388 Umekawa, T., Chegini, N., and Khan, S.R. (2003). Increased expression of monocyte 389 chemoattractant protein-1 (MCP-1) by renal epithelial cells in culture on exposure 390 to calcium oxalate, phosphate and uric acid crystals. Nephrol Dial Transplant 18, 391 664-669.

392 
395 Table 1: Clinical characteristics of study subjects

\begin{tabular}{lllll}
\hline & 2008 & 2013 & t or $Z$ & $p$-value* \\
\hline Age (years) & $47.84 \pm 12.64$ & - & - \\
Gender & & & - \\
Male (\%) & $1791(69.31)$ & - & - \\
Female (\%) & $793(30.69)$ & - & - \\
Habitual drinker (\%) & $582(22.52)$ & - & - & - \\
Habitual smoker $(\%)$ & $572(22.14)$ & - & - & $<0.01$ \\
SUA (umol/L) & $322.00(382.00,267.00)$ & $352.00(410.00,295.00)$ & -22.43 & $<0.01$ \\
eGFR (mL/min/1.73m $\left.{ }^{2}\right)$ & $103.99(113.31,95.11)$ & $101.19(113.24,101.19)$ & -5.49 & 0.09 \\
BMI (kg/m $\left.{ }^{2}\right)$ & $24.86 \pm 3.24$ & $24.06 \pm 2.46$ & 1.68 & 0.75 \\
Systolic BP (mmHg) & $122.00(135.00,111.00)$ & $122.00(136.00,110.00)$ & -0.31 & $<0.01$ \\
Diastolic BP (mmHg) & $73.00(81.00,66.00)$ & $71.00(79.00,63.00)$ & -13.39 & $<0.01$ \\
FPG (mmol/L) & $4.99(5.47,4.67)$ & $5.32(5.83,4.93)$ & -23.95 & $<0.01$ \\
TC (mmol/L) & $5.14(5.77,4.49)$ & $5.19(5.84,4.61)$ & -5.70 & $<0.05$ \\
TG (mmol/L) & $1.25(1.93,0.84)$ & $1.30(1.87,0.92)$ & -2.23 & - \\
\hline
\end{tabular}

396 Data are presented as mean \pm SD for normally distributed data, median (Q75, Q25) for non-normally distributed data or $\mathrm{n}$ 397 (percentage) for categorical data.

$398 * 2008$ versus 2013.

399 SUA: serum uric acid; BMI, body mass index; FPG: fasting plasma glucose; TC: total cholesterol; TG: triglycerides. 
401 Table 2: Univariate linear regression analysis for change in eGFR from 2008 to 2013 402 ( $\triangle$ eGFR) and independent variables. 403

\begin{tabular}{lll}
\hline & $t^{\prime} / r$ & $p$ 值 \\
\hline Gender & 8.30 & $<0.01$ \\
Habitual drinker & -0.06 & 0.95 \\
Habitual smoker & -0.03 & 0.98 \\
SUA & 0.03 & 0.09 \\
BMI & 0.02 & 0.42 \\
Systolic BP & -0.003 & 0.87 \\
Diastolic BP & 0.01 & 0.52 \\
FPG & 0.15 & $<0.01$ \\
TC & 0.10 & $<0.01$ \\
TG & 0.09 & $<0.01$ \\
eGFR at 2008 & -0.30 & $<0.01$ \\
$\Delta$ eGFR & -0.37 & $<0.01$ \\
\hline
\end{tabular}

404

405 
406 Table3: Clinical characteristics of three groups at baseline (2008): subjects with an increased SUA from 2008, with unchanged 407 SUA, and with a decreased SUA during the same period.

\begin{tabular}{|c|c|c|c|c|c|}
\hline & $\begin{array}{l}\text { Increased } \\
\text { SUA }\end{array}$ & Unchanged SUA & Decreased SUA & $F / \chi^{2}$ & $p$-value \\
\hline Sex & & & & 1.41 & 0.49 \\
\hline Male & 1154 & 214 & 423 & & \\
\hline Female & 500 & 108 & 185 & & \\
\hline Age (years,2008) & $47.50 \pm 12.37$ & $47.76 \pm 12.95$ & $48.80 \pm 13.17$ & 2.18 & 0.11 \\
\hline Drink habit & & & & 2.27 & 0.32 \\
\hline Habitual & 379 & 62 & 141 & & \\
\hline Non-habitual & 1275 & 260 & 467 & & \\
\hline Smoke habit & & & & 1.46 & 0.48 \\
\hline Habitual & 378 & 69 & 125 & & \\
\hline Non-habitual & 1276 & 253 & 483 & & \\
\hline SUA (umol/L) & $310.00(403.00,253.00)$ & $315.00(377.00,267.00)$ & $377.50(428.50,318.0)$ & 284.40 & $<0.01$ \\
\hline BMI $\left(\mathrm{kg} / \mathrm{m}^{2}\right)$ & $24.65 \pm 3.18$ & $24.83 \pm 3.37$ & $25.28 \pm 3.27$ & 13.70 & $<0.01$ \\
\hline $\mathrm{SBP}(\mathrm{mmHg})$ & $121.00(135.00,110.00)$ & $119.00(132.00,110.0)$ & $125.00(138.00,114.00)$ & 17.78 & $<0.05$ \\
\hline DBP $\quad(\mathrm{mmHg})$ & $73.00(81.00,66.00)$ & $74.00(80.50,66.00)$ & $75.00(84.00,68.00)$ & 11.20 & $<0.01$ \\
\hline FPG (mmol/L) & $4.97(5.42,4.66)$ & $5.00(5.51,4.66)$ & $5.07(5.70,4.73)$ & 17.40 & $<0.01$ \\
\hline $\mathrm{TC}(\mathrm{mmol} / \mathrm{L})$ & $5.10(5.74,4.48)$ & $5.17(5.91,4.56)$ & $5.21(5.91,4.56)$ & 5.57 & 0.05 \\
\hline $\mathrm{TG}(\mathrm{mmol} / \mathrm{L})$ & $1.22(1.86,0.82)$ & $1.18(1.84,0.80)$ & $1.41(2.12,0.92)$ & 23.50 & $<0.01$ \\
\hline $\begin{array}{l}\text { eGFR at } 2008 \\
\left(\mathrm{~mL} / \mathrm{min} / 1.73 \mathrm{~m}^{2}\right)\end{array}$ & $105.16(113.78,96.23)$ & $103.69(112.59,95.97)$ & $101.87(112.21,91.36)$ & 29.55 & $<0.01$ \\
\hline $\begin{array}{l}\triangle \mathrm{eGFR} \\
\left(\mathrm{mL} / \mathrm{min} / 1.73 \mathrm{~m}^{2}\right)\end{array}$ & $-5.26(3.71,-12.44)$ & $0.00(9.93,-6.64)$ & $3.23(15.25,-4.63)$ & 191.39 & $<0.01$ \\
\hline
\end{tabular}

408 Data are presented as mean \pm SD for normally distributed data, median (Q75, Q25) for non-normally distributed data or n. 
410 Table 4: Multiple linear regression analysis for change in eGFR from 2008 to 2013 ( $\triangle$ eGFR)

411 and independent variables.

412

413

\begin{tabular}{lllll}
\hline & $\beta$ & SE & $t$ & $p$-value \\
\hline Intercept & 42.24 & 3.88 & 10.88 & $<0.01$
\end{tabular}

$\triangle$ SUA

$\begin{array}{lllll}\text { Increased SUA } & -11.52 & 0.90 & -12.79 & <0.01\end{array}$

Unchanged SUA $\quad-5.32$

1.24

$-4.28$

$<0.01$

Decreased SUA 0

0

SUA (umol/L) $\quad-0.03$

$<0.01$

$-5.37$

$<0.01$

$\mathrm{FPG}(\mathrm{mmol} / \mathrm{L})$

0.14

0.03

4.06

$<0.01$

$\mathrm{TC}(\mathrm{mmol} / \mathrm{L})$

0.83

0.38

2.15

$<0.05$

TG (mmol/L)

0.99

0.32

3.11

$<0.01$

eGFR

$-0.32$

0.02

$-13.87$

$<0.01$

$\left(\mathrm{mL} / \mathrm{min} / 1.73 \mathrm{~m}^{2}\right)$

$414 \quad \mathrm{R}^{2}=0.17$

$415 \beta$, standardized regression coefficient. SE, standard error.

416 
417 Figure 1: Relationship between changes in SUA from 2008 to 2013 and changes in eGFR 418 during the same period. $\triangle$ SUA, change in the serum uric acid level from 2008 to 2013;

$419 \Delta$ eGFR, change in estimated glomerular filtration rate from 2008 to 2013.

420

421

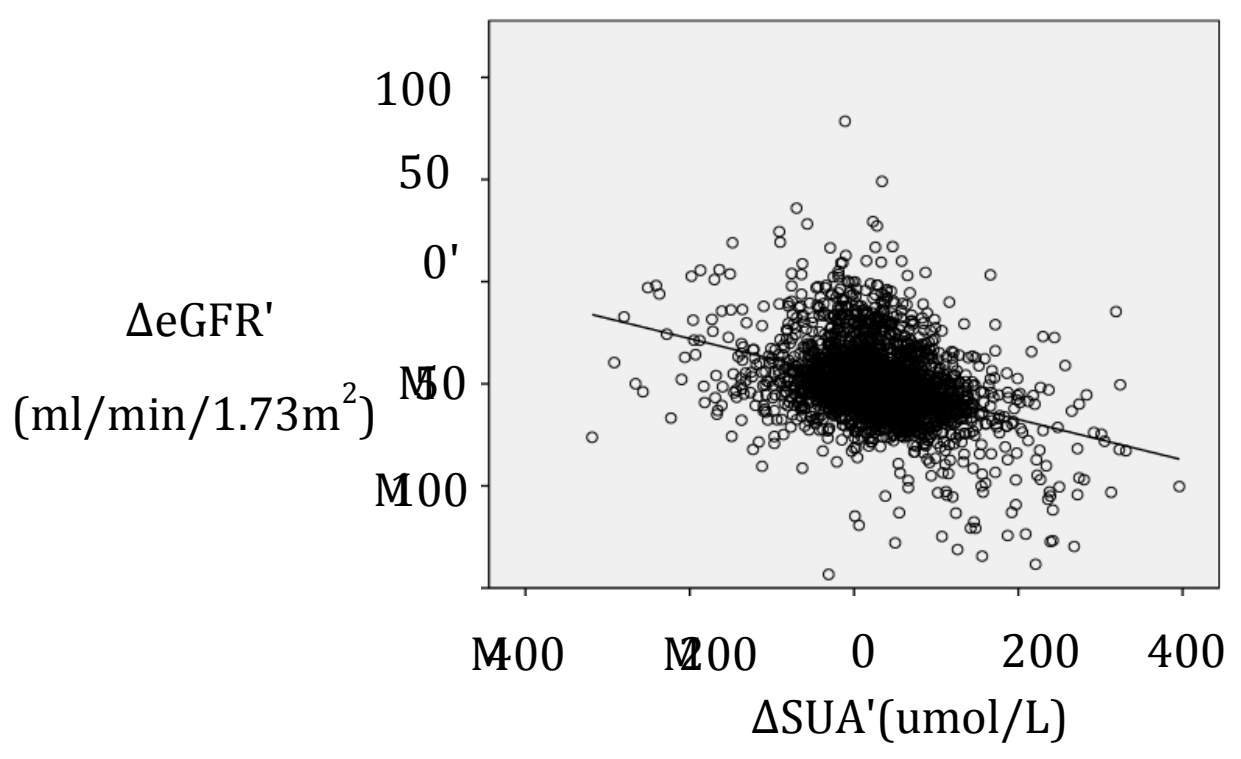

422

423

424

425

426

427

428

429

430

431

432

433

434

435

436 